\title{
Fragment-based discovery of novel allosteric MEK1 binders
}

Paolo Di Fruscia, ${ }^{a *}$ Fredrik Edfeldt, ${ }^{\text {b* }}$ Igor Shamovsky, ${ }^{\mathrm{c}}$ Gavin W. Collie, ${ }^{\mathrm{a}}$ Anna Aagaard, ${ }^{\mathrm{b}}$ Louise Barlind, ${ }^{\mathrm{d}}$ Ulf Börjesson, ${ }^{\mathrm{b}}$ Eva L. Hansson, ${ }^{\mathrm{e}}$ Richard J. Lewis, ${ }^{\mathrm{c}}$ Magnus K. Nilsson, ${ }^{\mathrm{c}}$ Linda Öster, ${ }^{\mathrm{b}}$ Josefine Pemberton, ${ }^{\mathrm{b}}$ Lena Ripa, ${ }^{\mathrm{c}} \mathrm{R}$. Ian Storer, ${ }^{\mathrm{a}}$ and Helena Käck ${ }^{\mathrm{b} *}$

${ }^{a}$ Structure Biophysics \& Fragments, Discovery Sciences, R\&D, AstraZeneca, Cambridge, UK.

${ }^{\mathrm{b} S}$ Structure Biophysics \& Fragments, Discovery Sciences, R\&D, AstraZeneca, Gothenburg, Sweden.

'Medicinal Chemistry, Research \& Early Development, Respiratory \& Immunology, BioPharmaceuticals R\&D, AstraZeneca, Gothenburg, Sweden.

dDiscovery Biology, Discovery Sciences, R\&D, AstraZeneca, Gothenburg, Sweden.

${ }^{e}$ Mechanistic Biology and Profiling, Discovery Sciences, R\&D, AstraZeneca, Gothenburg, Sweden.

\section{Supporting Information}

\section{Materials and Methods}

\section{Chemistry - General}

${ }^{1} \mathrm{H},{ }^{13} \mathrm{C}$ and ${ }^{19} \mathrm{~F}$ NMR spectra were recorded on a Bruker UltraShield Spectrometer $\left({ }^{1} \mathrm{H}\right.$ NMR, 300/400 $\mathrm{MHz} ;{ }^{13} \mathrm{C}$ NMR, $125 \mathrm{MHz} ;{ }^{19} \mathrm{~F}$ NMR, $\left.376 \mathrm{MHz}\right)$ at ambient temperature. Chemical shifts $(\delta)$ are quoted in parts per million $(\mathrm{ppm})$ and are referenced to a residual solvent peak: DMSO- $d_{6}\left(\delta_{\mathrm{H}}: 2.50, \delta \mathrm{c}: 39.52\right)$, $\mathrm{MeOH}-d_{4}\left(\delta_{\mathrm{H}}: 3.31\right)$. Coupling constants $(J)$ are quoted in Hertz $(\mathrm{Hz})$. LC-MS analyses were conducted using a ACQUITY LC-MS 2020 system with UV detection performed from 190 to $400 \mathrm{~nm}$. Purities of all assayed compounds were $\geq 95 \%$ as determined by LC-MS. All manipulations of air or moisture sensitive materials were carried out in oven- or flame-dried glassware under an inert atmosphere of nitrogen or argon. Syringes, which were used to transfer reagents and solvents, were purged with nitrogen prior to use. Reaction solvents were obtained anhydrous from commercial suppliers. All reagents were obtained from commercial suppliers and used without further purification unless indicated otherwise. 


\section{Chemistry - Synthetic Procedures and Compound Characterization}

\section{Dimethyl 2-bromobutanedioate (II).}

To a solution of 2-bromosuccinic acid (5.0 g, $25.38 \mathrm{mmol})$ and $\mathrm{K}_{2} \mathrm{CO}_{3}(10.5 \mathrm{~g}, 76.15 \mathrm{mmol})$ in DMF (3 $\mathrm{mL})$ was added iodomethane $(10.8 \mathrm{~g}, 76.15 \mathrm{mmol})$ at $0{ }^{\circ} \mathrm{C}$, and the reaction mixture stirred at room temperature for $3 \mathrm{~h}$. The solution was partitioned between EtOAc $(500 \mathrm{~mL})$ and $\mathrm{H}_{2} \mathrm{O}(150 \mathrm{~mL})$, the organic phase separated, washed with brine $(150 \mathrm{~mL})$, dried over anhydrous $\mathrm{Mg}_{2} \mathrm{SO}_{4}$ and the filtrate evaporated under reduced pressure to give the title compound as a yellow solid (3.0 g, $53 \%$ ), which was used in the next step without further purification: ${ }^{1} \mathrm{H}$ NMR $\left(300 \mathrm{MHz}, \mathrm{MeOH}-d_{4}\right) \delta 6.86(\mathrm{~s}, 2 \mathrm{H}), 3.83(\mathrm{~s}, 6 \mathrm{H})$.

\section{Methyl 2-(3-oxothiomorpholin-2-yl)acetate (IV).}

To a solution II (3.0 g, $14.84 \mathrm{mmol})$ in $\mathrm{MeOH}(70 \mathrm{~mL})$ was added 2-aminoethane-1-thiol (1.3 g, 16.33 mmol) at $0{ }^{\circ} \mathrm{C}$, and the reaction mixture stirred at room temperature for $2 \mathrm{~h} . \mathrm{K}_{2} \mathrm{CO}_{3}(4.7 \mathrm{~g}, 33.90 \mathrm{mmol})$ was then added and the resulting suspension stirred at $60{ }^{\circ} \mathrm{C}$ for $5 \mathrm{~h}$. The mixture was filtered and the filtrate evaporated under reduced pressure. The resultant residue was purified by silica gel flash-column chromatography, with $\mathrm{DCM} / \mathrm{MeOH}(8: 2)$ as the eluent, to give the title compound as a pale yellow solid (1.5 g, 59\% over 2 steps): ${ }^{1} \mathrm{H}$ NMR (300 MHz, DMSO-d 6$) \delta 8.02$ (s, 1H), $3.86(\mathrm{t}, J=7.0 \mathrm{~Hz}, 1 \mathrm{H}), 3.61$ (s, 3H), $3.49-3.42(\mathrm{~m}, 1 \mathrm{H}), 3.18(\mathrm{~d}, J=1.8 \mathrm{~Hz}, 2 \mathrm{H}), 2.92(\mathrm{dt}, J=11.8,4.0 \mathrm{~Hz}, 1 \mathrm{H}), 2.85-2.74(\mathrm{~m}, 2 \mathrm{H})$. LC-MS: $t_{\mathrm{R}}=0.49 \mathrm{~min}$; MS (ESI) $m / z$ calcd for $\mathrm{C}_{7} \mathrm{H}_{12} \mathrm{NO}_{3} \mathrm{~S}(\mathrm{M}+\mathrm{H})^{+}: 190.1$, found: 190.1 .

\section{2-(3-Oxothiomorpholin-2-yl)acetic acid (V).}

To a solution IV $(1.5 \mathrm{~g}, 7.93 \mathrm{mmol})$ in $\mathrm{MeOH}(5 \mathrm{~mL})$ was added $\mathrm{NaOH}(0.32 \mathrm{~g}, 7.93 \mathrm{mmol})$ at $0{ }^{\circ} \mathrm{C}$, and the reaction mixture stirred at room temperature for $3 \mathrm{~h}$. The solution was partitioned between EtOAc $(100 \mathrm{~mL})$ and aq. $\mathrm{HCl} 2 \mathrm{~N}(50 \mathrm{~mL})$, the organic phase separated, dried over anhydrous $\mathrm{Mg}_{2} \mathrm{SO}_{4}$ and the filtrate evaporated under reduced pressure to give the title compound as a pale yellow solid (0.8 g, 58\%), which was used in the next step without further purification: LC-MS: $t_{\mathrm{R}}=0.28 \mathrm{~min}$; MS (ESI) $\mathrm{m} / z \mathrm{calcd}$ for $\mathrm{C}_{6} \mathrm{H}_{10} \mathrm{NO}_{3} \mathrm{~S}(\mathrm{M}+\mathrm{H})^{+}:$176.0, found: 176.1 


\section{$N$-(4-methoxyphenyl)-2-(3-oxothiomorpholin-2-yl)acetamide (14).}

To a solution of $\mathbf{V}(0.5 \mathrm{~g}, 2.85 \mathrm{mmol})$ in DMF $(5 \mathrm{~mL})$ were added HATU (1.3 g, $3.42 \mathrm{mmol})$, DIPEA (1.3 $\mathrm{mL}, 7.13 \mathrm{mmol}$ ) and 4-methoxyaniline (422 $\mathrm{mg}, 3.42 \mathrm{mmol}$ ), and the reaction mixture stirred at room temperature for $3 \mathrm{~h}$. The solution was partitioned between EtOAc $(100 \mathrm{~mL})$ and water $(100 \mathrm{~mL})$, the organic phase separated, washed with brine $(100 \mathrm{~mL})$, dried over anhydrous $\mathrm{Mg}_{2} \mathrm{SO}_{4}$ and the filtrate evaporated under reduced pressure. The resultant residue was purified by preparative HPLC (XBridge Prep. C18 OBD Column, 5um,19*150mm; Mobile Phase A: $\mathrm{H}_{2} \mathrm{O}\left(10 \mathrm{mmol} / \mathrm{L} \mathrm{NH}_{4} \mathrm{HCO}_{3}\right)$, Mobile Phase B: ACN; Flow rate: $20 \mathrm{~mL} / \mathrm{min}$; Gradient: $16 \% \mathrm{~B}$ to $16 \% \mathrm{~B}$ in $16 \mathrm{~min} ; 220 / 254 \mathrm{~nm} ; t_{\mathrm{R}}: 14.20 \mathrm{~min}$ ). Fractions containing the desired compound were evaporated to dryness and combined to give the title copound as a white solid (39 mg, 5\%): ${ }^{1} \mathrm{H}$ NMR (400 MHz, DMSO- $\left.d_{6}\right) \delta 9.87(\mathrm{~s}, 1 \mathrm{H}), 7.95(\mathrm{t}, J=4.1 \mathrm{~Hz}, 1 \mathrm{H}), 7.54$ $7.40(\mathrm{~m}, 2 \mathrm{H}), 6.96-6.79(\mathrm{~m}, 2 \mathrm{H}), 3.93(\mathrm{t}, J=6.9 \mathrm{~Hz}, 1 \mathrm{H}), 3.72(\mathrm{~s}, 3 \mathrm{H}), 3.53-3.36(\mathrm{~m}, 2 \mathrm{H}), 2.96-2.87$ $(\mathrm{m}, 2 \mathrm{H}), 2.79(\mathrm{ddd}, J=11.8,10.0,5.1 \mathrm{~Hz}, 1 \mathrm{H}), 2.43(\mathrm{dd}, J=15.3,7.4 \mathrm{~Hz}, 1 \mathrm{H}) .{ }^{13} \mathrm{C} \mathrm{NMR}(125 \mathrm{MHz}$, DMSO- $\left.d_{6}\right) \delta 170.4$, 168.4, 155.6, 132.9, 121.0, 114.3, 55.6, 41.9, 37.6, 36.6, 27.2. LC-MS: $t_{\mathrm{R}}=1.73 \mathrm{~min}$; MS (ESI) $m / z$ calcd for $\mathrm{C}_{13} \mathrm{H}_{17} \mathrm{~N}_{2} \mathrm{O}_{3} \mathrm{~S}(\mathrm{M}+\mathrm{H})^{+}$: 281.1, found: 280.9. HRMS (ESI) $\mathrm{m} / z$ calcd for $\mathrm{C}_{13} \mathrm{H}_{17} \mathrm{~N}_{2} \mathrm{O}_{3} \mathrm{~S}(\mathrm{M}+\mathrm{H})^{+}: 281.0960$, found: 281.0966 .

Racemate $N$-(4-methoxyphenyl)-2-(3-oxothiomorpholin-2-yl)acetamide (14) was resolved by preparative chiral-HPLC (CHIRALPAK IA, 2*25cm,5um; Mobile Phase A = Hex:DCM 3/1, Mobile Phase B = EtOH; Flow rate: $17 \mathrm{~mL} / \mathrm{min}$; Gradient: 50\% B to 50\% B in $16.5 \mathrm{~min}$; 220/254 nm). Fractions containing the desired compounds were evaporated to dryness to afford ( $S$ )- $N$-(4-methoxyphenyl)-2-(3-oxothiomorpholin-2-yl)acetamide (23) as a white solid ( $\left.t_{\mathrm{R}}=8.16 \mathrm{~min} ; 60 \mathrm{mg}, 60 \%\right)$ and $(R)-N$-(4-methoxyphenyl)2-(3-oxothiomorpholin-2-yl)acetamide (24) as a white solid $\left(t_{\mathrm{R}}=12.87 \mathrm{~min} ; 40 \mathrm{mg}, 40 \%\right)$.

\section{(S)-N-(4-methoxyphenyl)-2-(3-oxothiomorpholin-2-yl)acetamide (23).}

${ }^{1} \mathrm{H}$ NMR (300 MHz, DMSO-d $) \delta 9.87(\mathrm{~s}, 1 \mathrm{H}), 7.95(\mathrm{~s}, 1 \mathrm{H}), 7.59$ - $7.39(\mathrm{~m}, 2 \mathrm{H}), 6.97$ - $6.72(\mathrm{~m}, 2 \mathrm{H})$, $3.93(\mathrm{t}, J=6.8 \mathrm{~Hz}, 1 \mathrm{H}), 3.73(\mathrm{~s}, 3 \mathrm{H}), 3.50(\mathrm{dq}, \mathrm{J}=14.7,4.7,4.3 \mathrm{~Hz}, 2 \mathrm{H}), 3.00-2.87$ (m, 2H), 2.80 (ddd, $J=11.8,10.0,5.1 \mathrm{~Hz}, 1 \mathrm{H}), 2.47-2.35(\mathrm{~m}, 1 \mathrm{H}) .{ }^{13} \mathrm{C}$ NMR $\left(125 \mathrm{MHz}, \mathrm{DMSO}-d_{6}\right) \delta 170.4,168.4,155.6$, 132.9, 121.0, 114.3, 55.6, 41.9, 37.6, 36.6, 27.2. LC-MS: $t_{\mathrm{R}}=2.21 \mathrm{~min}$; MS (ESI) $m / z$ calcd for $\mathrm{C}_{13} \mathrm{H}_{17} \mathrm{~N}_{2} \mathrm{O}_{3} \mathrm{~S}(\mathrm{M}+\mathrm{H})^{+}: 281.1$, found: 281.2 . HRMS (ESI) $m / z$ calcd for $\mathrm{C}_{13} \mathrm{H}_{17} \mathrm{~N}_{2} \mathrm{O}_{3} \mathrm{~S}(\mathrm{M}+\mathrm{H})^{+}: 281.0960$, found: 281.0966 . 


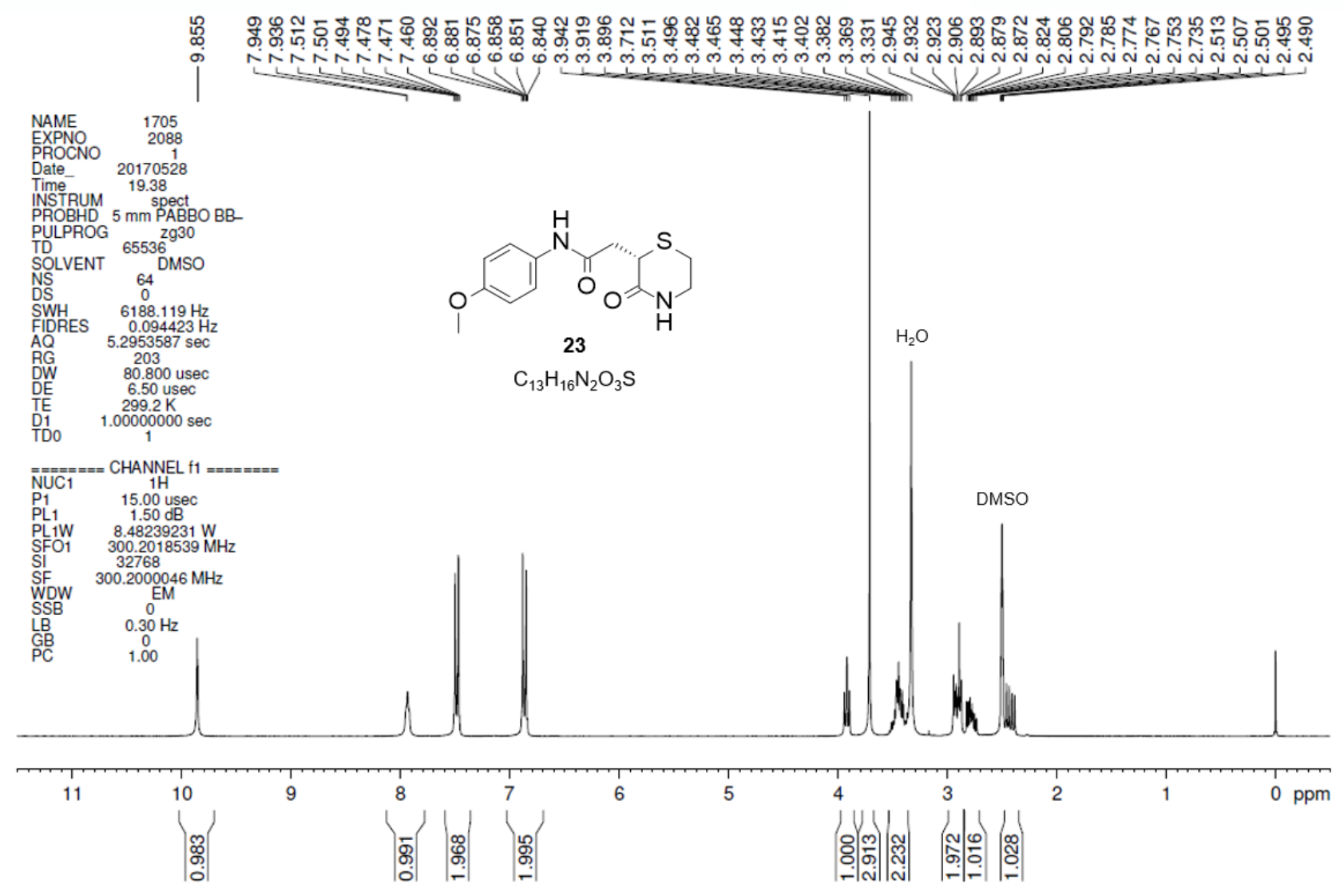

(R)-N-(4-methoxyphenyl)-2-(3-oxothiomorpholin-2-yl)acetamide (24).

${ }^{1} \mathrm{H}$ NMR (300 MHz, DMSO-d6) $\delta 9.87(\mathrm{~s}, 1 \mathrm{H}), 7.95(\mathrm{~s}, 1 \mathrm{H}), 7.56-7.43(\mathrm{~m}, 2 \mathrm{H}), 6.94-6.80(\mathrm{~m}, 2 \mathrm{H})$, $3.93(\mathrm{t}, J=6.9 \mathrm{~Hz}, 1 \mathrm{H}), 3.73(\mathrm{~s}, 3 \mathrm{H}), 3.46(\mathrm{tt}, J=9.5,4.0 \mathrm{~Hz}, 2 \mathrm{H}), 3.00-2.86(\mathrm{~m}, 2 \mathrm{H}), 2.84-2.70(\mathrm{~m}$, 1H), 2.44 (dd, $J=15.3,7.4 \mathrm{~Hz}, 1 \mathrm{H}) .{ }^{13} \mathrm{C}$ NMR (125 MHz, DMSO-d $) \delta$ 170.4, 168.4, 155.6, 132.9, 121.0, 114.3, 55.6, 41.9, 37.6, 36.6, 27.2. LC-MS: $t_{\mathrm{R}}=1.36 \mathrm{~min} ; \mathrm{MS}(\mathrm{ESI}) \mathrm{m} / z$ calcd for $\mathrm{C}_{13} \mathrm{H}_{17} \mathrm{~N}_{2} \mathrm{O}_{3} \mathrm{~S}(\mathrm{M}+\mathrm{H})^{+}$: 281.1, found: 281.2. HRMS (ESI) $m / z$ calcd for $\mathrm{C}_{13} \mathrm{H}_{17} \mathrm{~N}_{2} \mathrm{O}_{3} \mathrm{~S}(\mathrm{M}+\mathrm{H})^{+}: 281.0960$, found: 281.0966. 


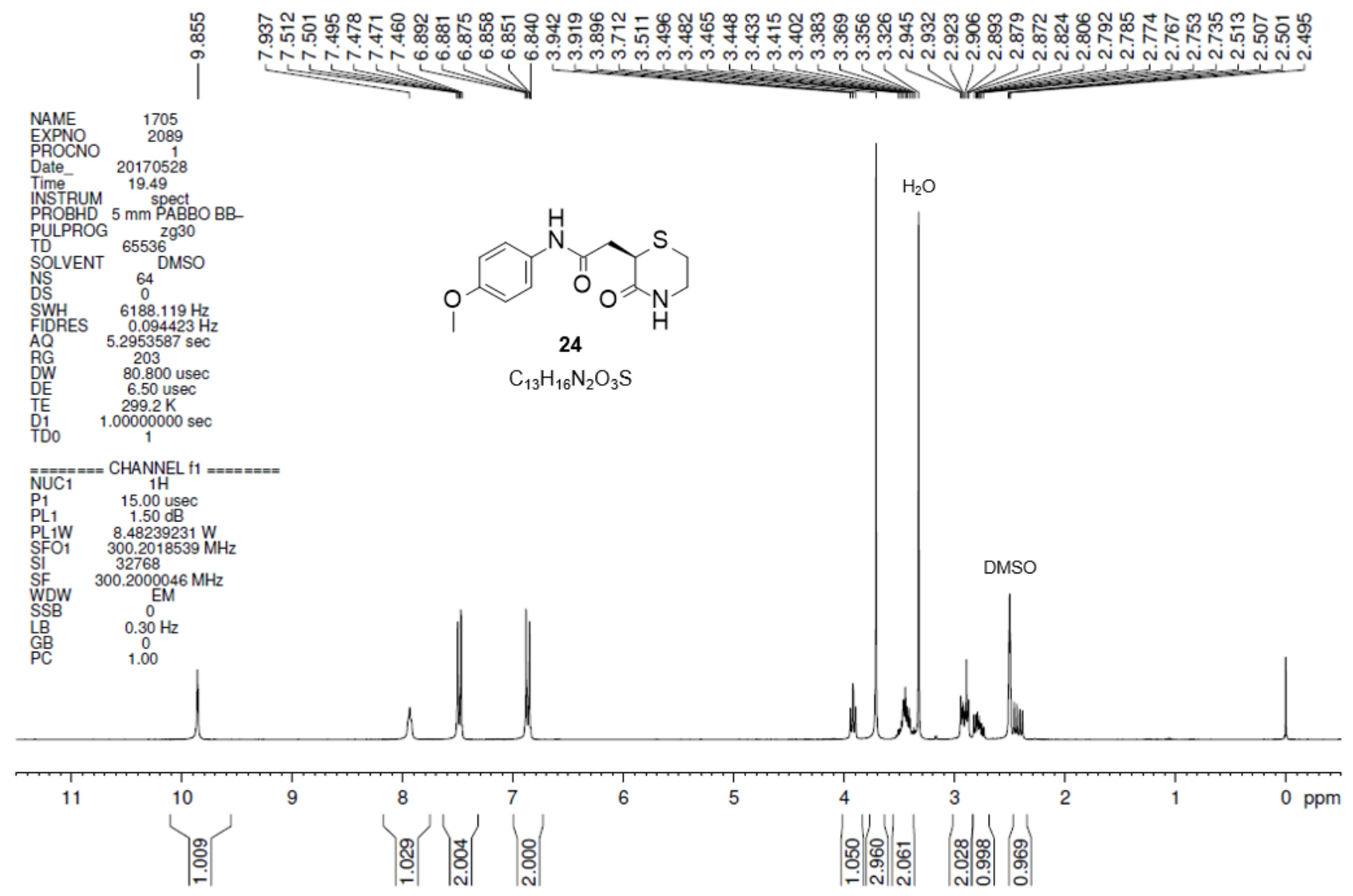

\section{2-(3-Oxothiomorpholin-2-yl)-N-phenyl-acetamide (11).}

${ }^{1} \mathrm{H}$ NMR (300 MHz, DMSO-d $) \delta 9.99(\mathrm{~s}, 1 \mathrm{H}), 7.93(\mathrm{~d}, J=5.4 \mathrm{~Hz}, 1 \mathrm{H}), 7.73-7.53(\mathrm{~m}, 2 \mathrm{H}), 7.43-7.24$ (m, 2H), $7.16-6.94(\mathrm{~m}, 1 \mathrm{H}), 3.94(\mathrm{t}, J=6.9 \mathrm{~Hz}, 1 \mathrm{H}), 3.56-3.34(\mathrm{~m}, 2 \mathrm{H}), 3.03-2.86(\mathrm{~m}, 2 \mathrm{H}), 2.79$ (ddd, $J=11.8,9.6,5.4 \mathrm{~Hz}, 1 \mathrm{H}), 2.49-2.43(\mathrm{~m}, 1 \mathrm{H})$. LC-MS: $t_{\mathrm{R}}=2.21 \mathrm{~min}$; MS $(\mathrm{ESI}) \mathrm{m} / z$ calcd for $\mathrm{C}_{12} \mathrm{H}_{15} \mathrm{~N}_{2} \mathrm{O}_{2} \mathrm{~S}(\mathrm{M}+\mathrm{H})^{+}: 251.1$, found: 251.1 . Yield of final amide coupling: $40 \%$.

\section{$N$-(2-methoxyphenyl)-2-(3-oxothiomorpholin-2-yl)acetamide (12).}

${ }^{1} \mathrm{H}$ NMR $\left(300 \mathrm{MHz}, \mathrm{DMSO}-d_{6}\right) \delta 9.24(\mathrm{~s}, 1 \mathrm{H}), 8.07-7.91(\mathrm{~m}, 2 \mathrm{H}), 7.04(\mathrm{~d}, J=2.1 \mathrm{~Hz}, 2 \mathrm{H}), 6.89$ (ddd, $J=7.9,6.3,2.5 \mathrm{~Hz}, 1 \mathrm{H}), 3.92(\mathrm{t}, J=6.7 \mathrm{~Hz}, 1 \mathrm{H}), 3.83(\mathrm{~s}, 3 \mathrm{H}), 3.56-3.35(\mathrm{~m}, 2 \mathrm{H}), 3.08-2.87(\mathrm{~m}, 2 \mathrm{H})$, $2.80(\mathrm{ddd}, J=11.9,9.5,5.3 \mathrm{~Hz}, 1 \mathrm{H}), 2.62(\mathrm{dd}, J=15.4,7.0 \mathrm{~Hz}, 1 \mathrm{H}) . \mathrm{LC}-\mathrm{MS}: t_{\mathrm{R}}=2.37 \mathrm{~min}$; MS (ESI) $m / z$ calcd for $\mathrm{C}_{13} \mathrm{H}_{17} \mathrm{~N}_{2} \mathrm{O}_{3} \mathrm{~S}(\mathrm{M}+\mathrm{H})^{+}: 281.1$, found: 281.1 . Yield of final amide coupling: 52\%. 


\section{$N$-(3-methoxyphenyl)-2-(3-oxothiomorpholin-2-yl)acetamide (13).}

${ }^{1} \mathrm{H}$ NMR $\left(300 \mathrm{MHz}, \mathrm{DMSO}-d_{6}\right) \delta 9.98(\mathrm{~s}, 1 \mathrm{H}), 7.94(\mathrm{~s}, 1 \mathrm{H}), 7.31(\mathrm{t}, J=2.2 \mathrm{~Hz}, 1 \mathrm{H}), 7.20(\mathrm{t}, J=8.1 \mathrm{~Hz}$, 1H), $7.15-7.06(\mathrm{~m}, 1 \mathrm{H}), 6.62(\mathrm{ddd}, J=8.2,2.5,1.1 \mathrm{~Hz}, 1 \mathrm{H}), 3.94(\mathrm{t}, J=6.9 \mathrm{~Hz}, 1 \mathrm{H}), 3.73(\mathrm{~s}, 3 \mathrm{H}), 3.60$ - $3.36(\mathrm{~m}, 2 \mathrm{H}), 3.02$ - $2.87(\mathrm{~m}, 2 \mathrm{H}), 2.79(\mathrm{ddd}, J=11.8,9.6,5.3 \mathrm{~Hz}, 1 \mathrm{H}), 2.48$ - 2.40 (m, 1H). LC-MS: $t_{\mathrm{R}}$ $=2.35 \mathrm{~min}$; MS (ESI) $\mathrm{m} / z$ calcd for $\mathrm{C}_{13} \mathrm{H}_{17} \mathrm{~N}_{2} \mathrm{O}_{3} \mathrm{~S}(\mathrm{M}+\mathrm{H})^{+}:$281.1, found: 281.2. Yield of final amide coupling: $39 \%$.

\section{$N$-(3-fluoro-4-methoxy-phenyl)-2-(3-oxothiomorpholin-2-yl)acetamide (15).}

${ }^{1} \mathrm{H}$ NMR $\left(400 \mathrm{MHz}, \mathrm{DMSO}-d_{6}\right) \delta 10.06(\mathrm{~s}, 1 \mathrm{H}), 7.97$ (s, 1H), 7.59 (dd, $\left.J=13.8,2.4 \mathrm{~Hz}, 1 \mathrm{H}\right), 7.23(\mathrm{~d}, J$ $=8.9 \mathrm{~Hz}, 1 \mathrm{H}), 7.11(\mathrm{t}, J=9.3 \mathrm{~Hz}, 1 \mathrm{H}), 3.93(\mathrm{t}, J=6.9 \mathrm{~Hz}, 1 \mathrm{H}), 3.80(\mathrm{~s}, 3 \mathrm{H}), 3.54-3.38(\mathrm{~m}, 2 \mathrm{H}), 2.91$ $(\mathrm{ddd}, J=15.4,7.9,5.3 \mathrm{~Hz}, 2 \mathrm{H}), 2.83-2.73(\mathrm{~m}, 1 \mathrm{H}), 2.44(\mathrm{dd}, J=15.4,7.2 \mathrm{~Hz}, 1 \mathrm{H}) .{ }^{19} \mathrm{~F} \mathrm{NMR}(376 \mathrm{MHz}$, DMSO- $\left.d_{6}\right) \delta$-134.0. LC-MS: $t_{\mathrm{R}}=2.21 \mathrm{~min}$; MS (ESI) $\mathrm{m} / z$ calcd for $\mathrm{C}_{13} \mathrm{H}_{16} \mathrm{FN}_{2} \mathrm{O}_{3} \mathrm{~S}(\mathrm{M}+\mathrm{H})^{+}: 299.1$, found: 299.2. Yield of final amide coupling: $1 \%$.

\section{$N$-[4-(difluoromethoxy)phenyl]-2-(3-oxothiomorpholin-2-yl)acetamide (17).}

${ }^{1} \mathrm{H}$ NMR (400 MHz, DMSO-d 6 ) $\delta 10.13(\mathrm{~s}, 1 \mathrm{H}), 7.98(\mathrm{~s}, 1 \mathrm{H}), 7.62$ (d, $\left.J=9.0 \mathrm{~Hz}, 2 \mathrm{H}\right), 7.11(\mathrm{~d}, J=9.0$ Hz, 2H), 3.94 (t, J=6.9 Hz, 1H), 3.55 - 3.39 (m, 2H), 3.37 (d, J=12.8 Hz, 1H), 2.99 - 2.87 (m, 2H), 2.79 (ddd, $J=11.7,10.1,5.2 \mathrm{~Hz}, 1 \mathrm{H}), 2.49-2.42$ (m, 1H). ${ }^{19} \mathrm{~F}$ NMR (376 MHz, DMSO-d6) $\delta-81.5$ (s, 2F). LC-MS: $t_{\mathrm{R}}=1.96 \mathrm{~min}$; MS (ESI) $m / z$ calcd for $\mathrm{C}_{13} \mathrm{H}_{15} \mathrm{~F}_{2} \mathrm{~N}_{2} \mathrm{O}_{3} \mathrm{~S}(\mathrm{M}+\mathrm{H})^{+}: 317.1$, found: 316.9. Yield of final amide coupling: $7 \%$.

\section{2-(3-Oxothiomorpholin-2-yl)- $N$-[4-(trifluoromethoxy)phenyl]acetamide (18).}

${ }^{1} \mathrm{H}$ NMR $\left(400 \mathrm{MHz}, \mathrm{DMSO}-d_{6}\right) \delta 10.24(\mathrm{~s}, 1 \mathrm{H}), 7.99(\mathrm{t}, J=4.3 \mathrm{~Hz}, 1 \mathrm{H}), 7.75-7.63$ (m, 2H), $7.32(\mathrm{~d}, J$ $=8.6 \mathrm{~Hz}, 2 \mathrm{H}), 3.95(\mathrm{t}, J=6.9 \mathrm{~Hz}, 1 \mathrm{H}), 3.55-3.38(\mathrm{~m}, 2 \mathrm{H}), 3.02-2.84(\mathrm{~m}, 2 \mathrm{H}), 2.79(\mathrm{ddd}, J=11.7,10.0$, $5.2 \mathrm{~Hz}, 1 \mathrm{H}), 2.47(\mathrm{~d}, J=7.1 \mathrm{~Hz}, 1 \mathrm{H}) .{ }^{19} \mathrm{~F}$ NMR $\left(376 \mathrm{MHz}, \mathrm{DMSO}-d_{6}\right) \delta-57.1(\mathrm{~s}, 3 \mathrm{~F})$. LC-MS: $t_{\mathrm{R}}=1.84$ min; MS (ESI) $m / z$ calcd for $\mathrm{C}_{13} \mathrm{H}_{14} \mathrm{~F}_{3} \mathrm{~N}_{2} \mathrm{O}_{3} \mathrm{~S}(\mathrm{M}+\mathrm{H})^{+}: 335.1$, found: 335.3 . Yield of final amide coupling: $24 \%$. 


\section{$N$-(4-hydroxyphenyl)-2-(3-oxothiomorpholin-2-yl)acetamide (19).}

${ }^{1} \mathrm{H}$ NMR $\left(400 \mathrm{MHz}, \mathrm{DMSO}-d_{6}\right) \delta 9.75(\mathrm{~s}, 1 \mathrm{H}), 9.16(\mathrm{~s}, 1 \mathrm{H}), 7.94(\mathrm{t}, J=4.2 \mathrm{~Hz}, 1 \mathrm{H}), 7.41-7.27(\mathrm{~m}, 2 \mathrm{H})$, $6.73-6.61(\mathrm{~m}, 2 \mathrm{H}), 3.91(\mathrm{t}, J=6.9 \mathrm{~Hz}, 1 \mathrm{H}), 3.52-3.38(\mathrm{~m}, 2 \mathrm{H}), 2.90(\mathrm{ddd}, J=15.3,7.9,5.2 \mathrm{~Hz}, 2 \mathrm{H})$, $2.78(\mathrm{ddd}, J=11.8,10.0,5.1 \mathrm{~Hz}, 1 \mathrm{H}), 2.41(\mathrm{dd}, J=15.3,7.5 \mathrm{~Hz}, 1 \mathrm{H}) . \mathrm{LC}-\mathrm{MS}: t_{\mathrm{R}}=1.12 \mathrm{~min}$; MS (ESI) $m / z$ calcd for $\mathrm{C}_{12} \mathrm{H}_{15} \mathrm{~N}_{2} \mathrm{O}_{3} \mathrm{~S}(\mathrm{M}+\mathrm{H})^{+}: 267.1$, found: 267.0 . Yield of final amide coupling: $57 \%$.

\section{$N$-(4-methoxyphenyl)-N-methyl-2-(3-oxothiomorpholin-2-yl)acetamide (25).}

${ }^{1} \mathrm{H}$ NMR $\left(400 \mathrm{MHz}, \mathrm{DMSO}-d_{6}\right) \delta 7.88(\mathrm{~s}, 1 \mathrm{H}), 7.27(\mathrm{~d}, J=8.8 \mathrm{~Hz}, 2 \mathrm{H}), 7.01(\mathrm{~d}, J=8.8 \mathrm{~Hz}, 2 \mathrm{H}), 3.87$ (t, $J=6.7 \mathrm{~Hz}, 1 \mathrm{H}), 3.78(\mathrm{~s}, 3 \mathrm{H}), 3.47-3.34(\mathrm{~m}, 2 \mathrm{H}), 3.12(\mathrm{~s}, 3 \mathrm{H}), 2.85(\mathrm{dt}, J=11.6,3.8 \mathrm{~Hz}, 1 \mathrm{H}), 2.73-2.52$ $(\mathrm{m}, 2 \mathrm{H}), 2.07(\mathrm{dd}, J=16.0,6.9 \mathrm{~Hz}, 1 \mathrm{H})$. LC-MS: $t_{\mathrm{R}}=1.75 \mathrm{~min}$; MS (ESI) $\mathrm{m} / z$ calcd for $\mathrm{C}_{14} \mathrm{H}_{19} \mathrm{~N}_{2} \mathrm{O}_{3} \mathrm{~S}$ $(\mathrm{M}+\mathrm{H})^{+}:$295.1, found: 295.0. Yield of final amide coupling: $6 \%$.

\section{2-(4-Iodophenyl)-1H-imidazo[1,2-c]pyrimidin-5-one (9).}

To a solution of 2-bromo-1-(4-iodophenyl)ethanone $(300 \mathrm{mg}, 0.92 \mathrm{mmol})$ in EtOH (10 mL) were added 6-amino-1H-pyrimidin-2-one (113 mg, $1.02 \mathrm{mmol})$ and $\mathrm{NaHCO}_{3}(155 \mathrm{mg}, 1.85 \mathrm{mmol})$, and the reaction mixture stirred at room temperature for $2 \mathrm{~h}$. The solvent was evaporated under reduced pressure and the residue partitioned between EtOAc $(20 \mathrm{~mL})$ and $\mathrm{H}_{2} \mathrm{O}(20 \mathrm{~mL})$. The organic phase was separated, washed with brine $(20 \mathrm{~mL})$, dried over anhydrous $\mathrm{Mg}_{2} \mathrm{SO}_{4}$ and the filtrate evaporated under reduced pressure. The resultant residue was purified by preparative HPLC (XBridge Prep. OBD C18 Column, 5um, 30*150mm; Mobile Phase A: $\mathrm{H}_{2} \mathrm{O}\left(0.05 \% \mathrm{NH}_{3} \mathrm{H}_{2} \mathrm{O}\right)$, Mobile Phase B: ACN; Flow rate: $60 \mathrm{~mL} / \mathrm{min}$; Gradient: $30 \%$ B to $45 \%$ B in $7 \mathrm{~min} ; 220 / 254 \mathrm{~nm}$; $t_{\mathrm{R}}: 6.87 \mathrm{~min}$ ). Fractions containing the desired compound were evaporated to dryness and combined to give the title copound as a white solid (40 $\mathrm{mg}, 13 \%$ ): ${ }^{1} \mathrm{H}$ NMR (300 MHz, DMSO- $\left.d_{6}\right) \delta 11.61$ (br s, 1H), 8.36 (d, $\left.J=0.7 \mathrm{~Hz}, 1 \mathrm{H}\right), 7.79$ (app-d, J=0.9 Hz, 4H), $7.29(\mathrm{~d}, J=7.5 \mathrm{~Hz}, 1 \mathrm{H}), 6.61(\mathrm{dd}, J=7.5,0.7 \mathrm{~Hz}, 1 \mathrm{H})$. LC-MS: $t_{\mathrm{R}}=1.48 \mathrm{~min}$; MS (ESI) $\mathrm{m} / z$ calcd for $\mathrm{C}_{12} \mathrm{H}_{9} \mathrm{IN}_{3} \mathrm{O}(\mathrm{M}+\mathrm{H})^{+}: 337.9$, found: 338.0 . 


\section{Protein Expression and Purification}

The NMR and SPR assays were performed using human Avi-TEV-6His-MEK1 (full-length) expressed in $E$. coli. Cell pellet from E. coli was resuspended in $25 \mathrm{mM}$ Tris-HCl, pH 7.5, $500 \mathrm{mM} \mathrm{NaCl}, 10 \%$ glycerol, $1 \mathrm{mM}$ TCEP and protease inhibitor tablets (Complete EDTA-free) and lysed by sonication. The cleared supernatant was supplemented with $20 \mathrm{mM}$ imidazole and then loaded on to a column (HisTrap FF crude) equilibrated in buffer A (Buffer A: 25 mM Tris-HCl, pH 7.5, 500 mM NaCl, 10\% glycerol, 1 $\mathrm{mM}$ TCEP, $20 \mathrm{mM}$ imidazole). The protein was then eluted with buffer B (Buffer B: $25 \mathrm{mM}$ Tris- $\mathrm{HCl}$, pH 7.5, $500 \mathrm{mM} \mathrm{NaCl}, 10 \%$ glycerol, $1 \mathrm{mM}$ TCEP, $300 \mathrm{mM}$ imidazole). The pooled fractions were diluted 18 times in Buffer C (25 mM Tris-HCl, pH 7.5, 5\% glycerol, $1 \mathrm{mM}$ TCEP) and then loaded on to a ResourceQ column. The protein was eluted with a $20 \mathrm{CV}$ gradient with buffer D (25 mM Tris- $\mathrm{HCl}, \mathrm{pH}$ 7.5, $1 \mathrm{M} \mathrm{NaCl}, 5 \%$ glycerol, $1 \mathrm{mM}$ TCEP). The eluted protein was pooled followed by SEC gel filtration (Superdex 75 16/60) in the final buffer (25 mM Tris-HCl, pH 7.5, $150 \mathrm{mM} \mathrm{NaCl,} \mathrm{5 \%} \mathrm{glycerol,} 1 \mathrm{mM}$ TCEP). The protein for SPR was auto-biotinylated as assessed by mass spectrometry.

For crystallographic studies, human 6xHis-TEV-MEK1 (encompassing residues 37-383 with residues 264-307 replaced by GSGSGS) was expressed in Spodoptera frugiperda insect cells. For this, recombinant baculoviruses were generated and propagated using the Bac-to-Bac system. For expression, Spodoptera frugiperda (Sf21) insect cells were cultured in Sf-900 II SFM medium at $27^{\circ} \mathrm{C}$ and infected with baculovirus at a density of 2.5-3.0 $\times 10^{6} \mathrm{ml}^{-1}$. The culture was incubated for $48 \mathrm{~h}$ after infection, and cells were collected and washed with ice-cold Sf-900 II SFM medium, flash frozen in liquid $\mathrm{N}_{2}$ and stored at $80^{\circ} \mathrm{C}$. Cell pellet was resuspended in $25 \mathrm{mM}$ Tris-HCl, pH 7.5, $500 \mathrm{mM} \mathrm{NaCl}, 10 \%$ glycerol, $1 \mathrm{mM}$ TCEP and protease inhibitor tablets (Complete EDTA-free) and lysed by sonication. The cleared supernatant was supplemented with $20 \mathrm{mM}$ imidazole and then loaded on to a column (HisTrap HP) equilibrated in buffer A (Buffer A: 25 mM Tris-HCl, pH 8.7, 500 mM NaCl, 10\% glycerol, $1 \mathrm{mM} \mathrm{TCEP,} 20$ $\mathrm{mM}$ imidazole). The protein was then eluted with buffer B (Buffer B: $25 \mathrm{mM}$ Tris-HCl, pH 8.7, $500 \mathrm{mM}$ $\mathrm{NaCl}, 10 \%$ glycerol, $1 \mathrm{mM}$ TCEP, $300 \mathrm{mM}$ imidazole). The protein was further concentrated (3K, Amicon) followed by SEC gel filtration (Superdex 75 26/60) in the final buffer (25 mM Tris-HCl, $\mathrm{pH}$ 8.6, $150 \mathrm{mM} \mathrm{NaCl}$, 5\% glycerol, $1 \mathrm{mM}$ TCEP). Typical yield was $40 \mathrm{mg}$ from $1 \mathrm{~L}$ of Sf21 culture. 


\section{NMR Screening Assay}

A $1 \mathrm{D}{ }^{1} \mathrm{H}-\mathrm{NMR}$ direct binding assay was used to detect the binding of fragments to full-length MEK1. For these experiments, $1 \mathrm{mM}$ AMP-PNP was added in order to block the ATP-binding site. Addition of binimetinib and detection of any competition was performed to assess whether fragments were binding to the allosteric site of MEK1. Experiments were performed at $20{ }^{\circ} \mathrm{C}$ using standard CPMG experiments on a Bruker $600 \mathrm{MHz}$ spectrometer coupled to a TECAN sample preparation robot via a SampleRail. Buffer conditions were $50 \mathrm{mM}$ deuterated TRIS pH 7.5, $150 \mathrm{mM} \mathrm{NaCl}, 10 \% \mathrm{D}_{2} \mathrm{O}, 1 \mathrm{mM}$ TCEP, $1 \mathrm{mM}$ AMPPNP, $10 \mathrm{mM} \mathrm{MgCl} 2$ and $11 \mu \mathrm{M}$ TMSP (for chemical shift referencing). Samples were prepared just prior to measurement. For each fragment mixture three spectra were recorded: 1) mixture containing six fragments at $50 \mu \mathrm{M}$ nominal concentration, 2) with $7.5 \mu \mathrm{M}$ MEK1 protein added, 3) with $15 \mu \mathrm{M}$ binimetinib added. Fragments were considered as hits only if both binding and subsequent competition could be observed.

\section{SPR Screening Assay}

A streptavidin-coated sensor chip (GE Healthcare) was docked into a Biacore T200 or S200 instrument. The running buffer was $50 \mathrm{mM}$ HEPES pH 7.4, $150 \mathrm{mM} \mathrm{NaCl}, 10 \mathrm{mM} \mathrm{MgCl}, 0.005 \%$ (v/v) Tween20, $5 \mathrm{mM}$ DTT. The surface was prepared by an initial wash with $50 \mathrm{mM} \mathrm{NaOH} / 1 \mathrm{M} \mathrm{NaCl}$. Full-length biotinylated MEK1 at a concentration of $\sim 1 \mu \mathrm{M}$ was then injected over flow-cells 2 and 4 (leaving flowcells 1 and 3 for reference subtraction) with a contact time of 300-600 sec at a flow-rate of $10 \mu 1 / \mathrm{min}$. Typical immobilization levels were 6000-8000 response units (RU). As a last step, PEG-biotin was in-

jected over all flow-cells to saturate remaining streptavidin sites. Compounds were diluted either from $10 \mathrm{mM}$ or $100 \mathrm{mM}$ DMSO stocks to obtain a top concentration of $100 \mu \mathrm{M}$ or $1000 \mu \mathrm{M}$. 3-fold serial dilutions were then made to obtain 7 or 10 dose-response series. $1 \mathrm{mM}$ AMP-PNP was added to each well when determining affinities in the presence AMP-PNP. Surface activity was monitored by including the reference compound binimetinib first and last in each run. The assay was typically stable over several days at room temperature. Data were analyzed using GeneData software and $\mathrm{K}_{d}$ values determined using steady-state fits. 


\section{Crystallography}

Human MEK1 (residues 37-383 with residues 264-307 replaced with: GSGSGS) with an N-terminal 6xHis-tag was used for crystallisation. MEK1 was prepared in a buffer containing $25 \mathrm{mM}$ Tris $\mathrm{pH} 7.5$, $150 \mathrm{mM} \mathrm{NaCl}, 5 \%$ glycerol and $1 \mathrm{mM}$ TCEP at a concentration of $10 \mathrm{mg} / \mathrm{ml}$, mixed with $2 \mathrm{mM} \mathrm{MgCl}_{2}$ and $2 \mathrm{mM}$ AMP-PNP, and incubated at room temperature for 30 minutes. Crystals were obtained from

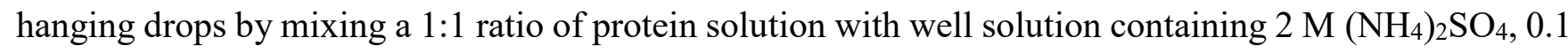
$\mathrm{M} \mathrm{NaCl}$ and 0.1 M Bis-tris pH 6.1-6.2 at room temperature. The structures of $\mathbf{6 , 9} 9$ and 23 were obtained by soaking crystals with $13 \mathrm{mM}$ of compound (100 mM in DMSO) overnight. Prior to flash freezing, the crystals were quickly dipped in a cryo solution consisting of $3 \mathrm{M}\left(\mathrm{NH}_{4}\right)_{2} \mathrm{SO}_{4}, 0.1 \mathrm{M} \mathrm{NaCl}$ and $0.1 \mathrm{M}$ Bistris $\mathrm{pH}$ 6.2. For 6 data were collected using a Rigaku FRE+ rotating anode with a CCD-A200-CU detector, for 9 data were collected at beamline ID30B at the European Synchrotron Radiation Facility (ESRF) in France, while data for 23 were collected at the BioMAX beamline at MAX IV in Lund, Sweden. The structure of 4 was obtained from co-crystallisation. For this, MEK1 was incubated with $2 \mathrm{mM} \mathrm{MgCl}_{2}, 2$ mM AMP-PNP and $5 \mathrm{mM} 4$ for 1 and $1 / 2$ hours prior to crystallisation. Crystals were obtained in $2.2 \mathrm{M}$ $\left(\mathrm{NH}_{4}\right)_{2} \mathrm{SO}_{4}, 0.1 \mathrm{M} \mathrm{NaCl}$ and $0.1 \mathrm{M}$ Tris $\mathrm{pH}$ 7. Prior to flash freezing, the crystals were briefly transferred to a cryo solution consisting of $3 \mathrm{M}\left(\mathrm{NH}_{4}\right)_{2} \mathrm{SO}_{4}, 0.1 \mathrm{M} \mathrm{NaCl}$ and $0.1 \mathrm{M}$ Tris $\mathrm{pH}$ 7. Data were collected at the ID29 beamline at the ESRF. Data were integrated and processed using autoPROC. ${ }^{1}$ The crystals belong to space group P $2{ }_{1} 2_{1} 2_{1}$ with two molecules in the asymmetric unit. The structures were solved by molecular replacement using the Protein Data Bank (PDB) entry 3E8N as search model. ${ }^{2}$ Structural refinement was carried out using AutoBuster ${ }^{3}$. Manual model building was performed using Coot. ${ }^{4}$ Ligand restraint dictionaries were generated using the program Writedict. ${ }^{5}$ All structure illustrations were prepared using the program Pymol. ${ }^{6}$ Crystallographic coordinates have been deposited in the Protein Data Bank with accession codes provided in Table S1 along with data collection and refinement statistics. 
Table S1. Statistics from crystallographic data reduction and refinement. Values in parenthesis refers to data in the highest resolution shell.

\begin{tabular}{|c|c|c|c|c|}
\hline Compound & 4 & 6 & 9 & 23 \\
\hline PDB accession code & 7B7R & $7 \mathrm{~B} 3 \mathrm{M}$ & 7B94 & 7B9L \\
\hline x-ray source/synchrotron & ID29/ESRF & FRE+/in-house & ID30B/ESRF & BioMAX/MAXlab \\
\hline Space group: & $\mathrm{P} 2{ }_{1} 2_{1} 2_{1}$ & $\mathrm{P} 2{ }_{1} 2_{1} 2_{1}$ & $\mathrm{P} 2{ }_{1} 2_{1} 2_{1}$ & $\mathrm{P} 2{ }_{1} 2_{1} 2_{1}$ \\
\hline Cell constants: a b c $(\AA)$ & 57.6671 .67157 .95 & 57.6871 .92157 .71 & 57.2871 .75157 .8 & 57.9572 .08158 .16 \\
\hline Resolution range $(\AA)$ & $54.1-1.7$ & $157.7-2.3$ & $78.9-2.00$ & $79.1-1.7$ \\
\hline Highest resolution shell ( $\AA$ ) & $1.74-1.7$ & $2.38-2.3$ & $2.04-2.00$ & $1.74-1.7$ \\
\hline Completeness overall (\%) & $99.9(99.8)$ & $93.8(99.3)$ & $99.9(97.2)$ & $99.4(93.0)$ \\
\hline Total number of observations & 458594 & 116570 & 268154 & 473128 \\
\hline Reflections, unique & 71615 & 28130 & 43278 & 74057 \\
\hline Redundancy & $6.4(4.7)$ & $4.1(4.2)$ & $6.2(6.3)$ & $6.4(4.5)$ \\
\hline$R$ merge overall ${ }^{1}$ & $0.040(0.673)$ & $0.111(0.463)$ & $0.064(0.76)$ & $0.039(0.604)$ \\
\hline $\mathrm{I} / \mathrm{SigI}^{2}$ & $21.7(2.1)$ & $12(3.5)$ & $15.3(2.1)$ & $20.9(1.8)$ \\
\hline $\mathrm{CC} 1 / 2$ & $0.99(0.77)$ & $0.89(0.80)$ & $0.99(0.86)$ & $0.99(0.79)$ \\
\hline$R$ value overall $(\%)^{3}$ & 19.4 & 21.7 & 21.6 & 19.3 \\
\hline$R$ value free $(\%)^{4}$ & 21.8 & 26.8 & 26.5 & 21.4 \\
\hline \multicolumn{5}{|c|}{ R.m.s. deviations from ideal values } \\
\hline Bond lengths $(\AA)$ & 0.010 & 0.009 & 0.010 & 0.010 \\
\hline Bond angles $\left({ }^{\circ}\right)$ & 1.09 & 1.14 & 1.20 & 1.06 \\
\hline \multicolumn{5}{|c|}{$\Phi, \Psi$ angle distribution for residues ${ }^{5}$} \\
\hline In most favoured regions (\%) & 94.8 & 93.6 & 94.2 & 95.7 \\
\hline $\begin{array}{l}\text { In additional allowed regions } \\
(\%)\end{array}$ & 4.8 & 5.8 & 5.4 & 3.7 \\
\hline In generously regions (\%) & 0.4 & 0.6 & 0.4 & 0.6 \\
\hline In disallowed regions (\%) & 0.0 & 0.0 & 0.0 & 0.0 \\
\hline
\end{tabular}

${ }^{1} R_{\text {merge }}=\Sigma_{h k l}\left[\left(\Sigma_{i}\left|I_{i}-\langle I\rangle\right|\right) / \Sigma_{i} I_{i}\right]$

${ }^{2} \mathrm{I} / \mathrm{sigI}$ avg is the mean $\mathrm{I} / \mathrm{sig}$ for the unique reflections in the output file

${ }^{3} R_{\text {value }}=\sum_{h k l}|| F_{\text {obs }}|-| F_{\text {calc }}|| / \sum_{h k l}\left|F_{\text {obs }}\right|$

${ }^{4} R_{\text {free }}$ is the cross-validation $R$ factor computed for the test set of $5 \%$ of unique reflections

${ }^{5}$ Ramachandran statistics as defined by PROCHECK ${ }^{7}$ 


\section{Vibrational Circular Dichroism (VCD)}

The separated enantiomers 23 and 24 were dissolved at a concentration of $0.154 \mathrm{M}$ in DMSO-d6. The solutions were transferred separately to a $0.0995 \mathrm{~mm} \mathrm{BaF}_{2}$ cell and VCD spectra acquired for ten hours in a Biotools ChiralIR2X instrument at a resolution of $4 \mathrm{~cm}^{-1}$ and PEM setting of $1400 \mathrm{~cm}^{-1}$. A blank spectrum of DMSO-d6 was also acquired. Infra-red spectra were acquired on the samples concomitantly. The experimental infra-red and VCD spectra are shown in Figures S1 and S2 respectively.

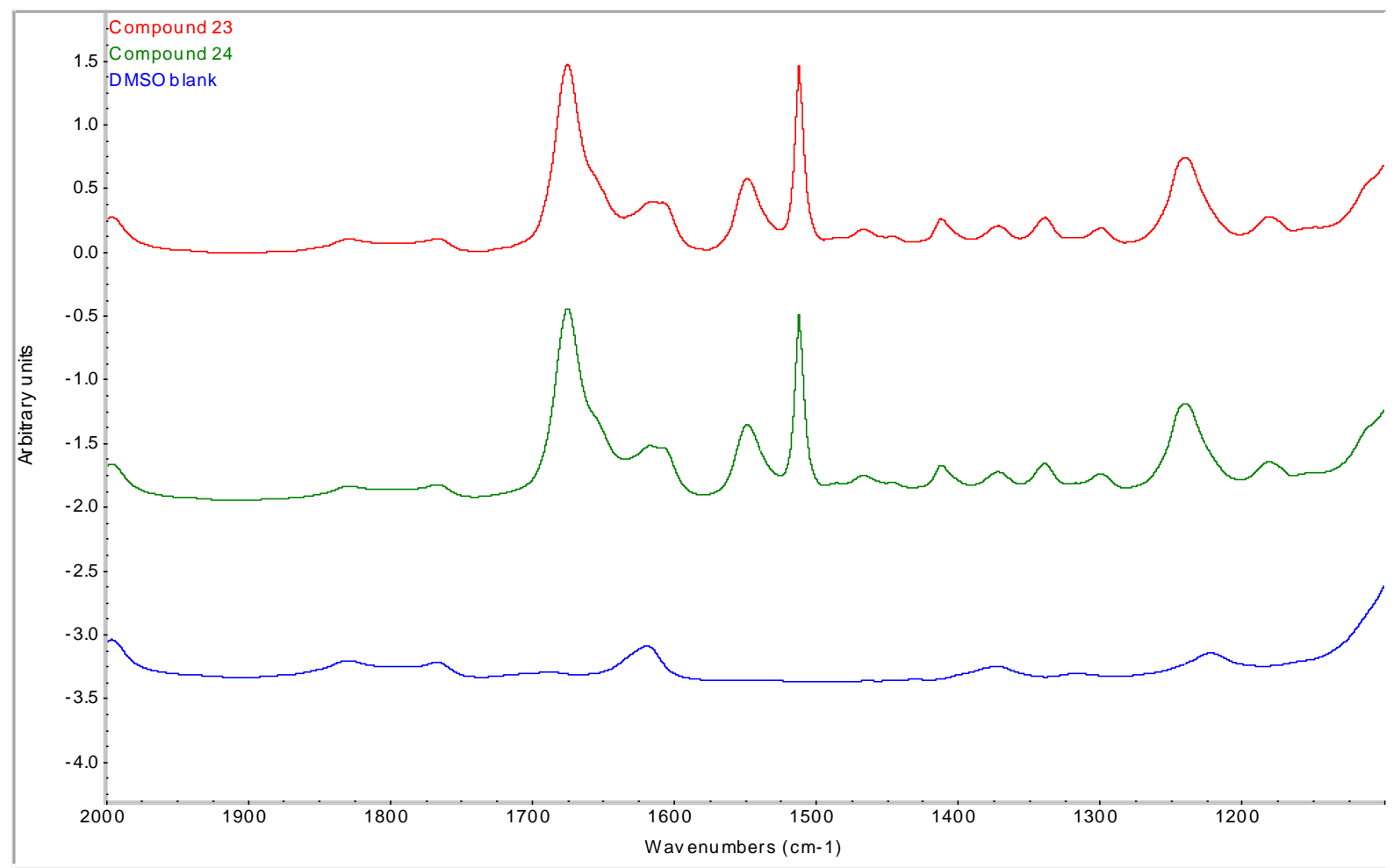

Figure S1. Experimental infra-red spectra for compounds 23 (red) and 24 (green) and blank DMSO-d6 (blue). 


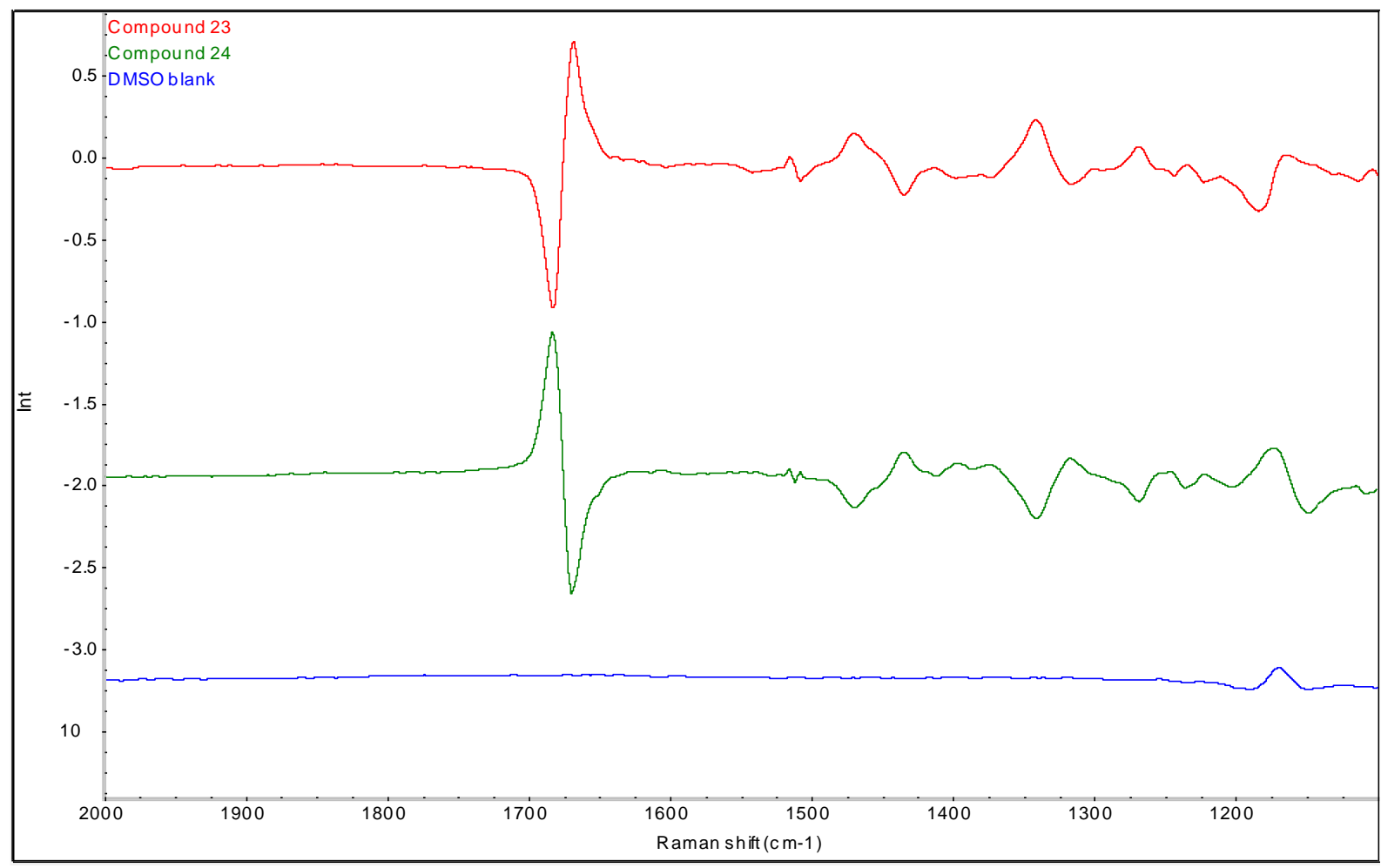

Figure S2. Experimental VCD spectra for compounds 23 (red) and 24 (green) and blank DMSO-d6 (blue).

\section{Computational Spectral Simulations}

A Monte Carlo molecular mechanics search for low energy geometries was conducted for the $S$ enantiomer. MacroModel within the Maestro graphical interface (Schrödinger Inc.) was used to generate 96 starting coordinates for conformers. All conformers within $21 \mathrm{kcal} / \mathrm{mole}$ of the lowest energy conformer were used as starting point for density functional theory (DFT) minimizations within Gaussian16. ${ }^{8}$ Optimized structures, harmonic vibrational frequencies/intensities, VCD rotational strengths, and free energies at STP (including zero-point energies) were determined in at the B3PW91/cc-pVTZ level of theory using the default polarizable continuum model for DMSO.

The minimum energy conformation found is shown in Figure S3 together with an overlay of the additional 13 conformations within $5 \mathrm{kJmol}^{-1}$ of this. The coordinates for the minimum energy conformation are shown in Table S2. An in-house program was used to generate a Boltzmann weighted average spectrum for these 14 conformations and fit Lorentzian line shapes $\left(12 \mathrm{~cm}^{-1}\right.$ line width) to the computed spectra applying a linear scaling factor of 0.98 . 

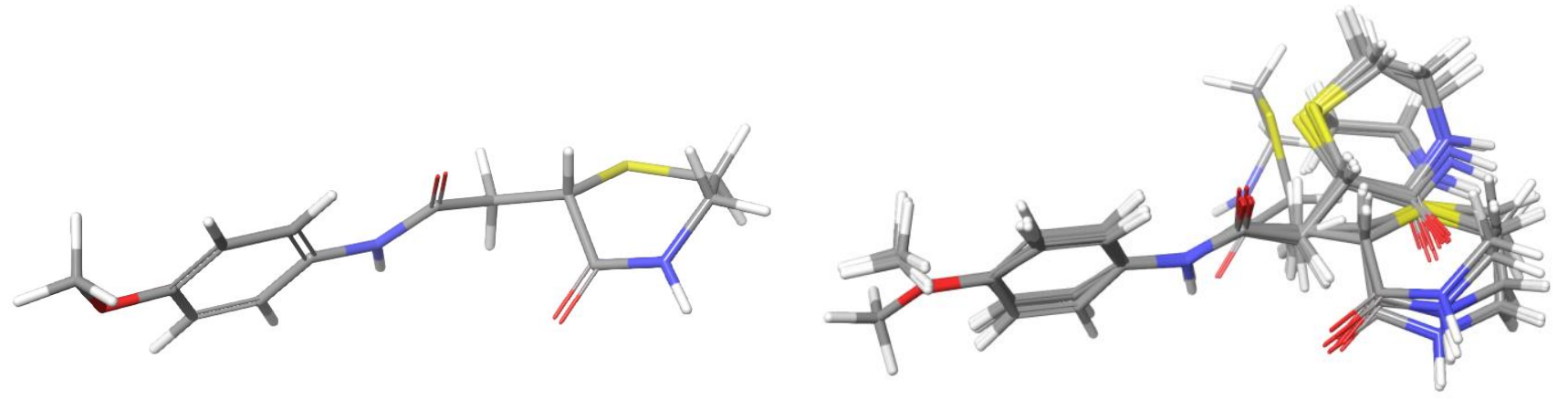

Figure S3. The minimum energy conformation (left) and an overlay of this with 13 additional conformations within $5 \mathrm{kJmol}^{-1}$ used in the calculation of the Boltzmann average infra-red and VCD spectra.

Table S2. Coordinates for the minimum energy conformation.

$\begin{array}{lrrr}\mathrm{C} & -2.12220 & -0.60690 & 0.06600 \\ \mathrm{C} & -2.63900 & 0.49450 & -0.60920 \\ \mathrm{C} & -4.00190 & 0.77320 & -0.56560 \\ \mathrm{C} & -4.86900 & -0.04240 & 0.15320 \\ \mathrm{C} & -4.35090 & -1.14620 & 0.83340 \\ \mathrm{C} & -3.00020 & -1.42170 & 0.78920 \\ \mathrm{~N} & -0.76200 & -0.96440 & 0.06690 \\ \mathrm{H} & -1.97740 & 1.13560 & -1.16900 \\ \mathrm{H} & -4.36900 & 1.63660 & -1.10220 \\ \mathrm{O} & -6.20940 & 0.14880 & 0.25210 \\ \mathrm{H} & -5.02350 & -1.78220 & 1.39470 \\ \mathrm{H} & -2.61750 & -2.28420 & 1.32320 \\ \mathrm{H} & -0.54540 & -1.79950 & 0.58700 \\ \mathrm{C} & 0.29120 & -0.35100 & -0.52980 \\ \mathrm{O} & 0.21080 & 0.68540 & -1.17470 \\ \mathrm{C} & 1.60550 & -1.10220 & -0.38180 \\ \mathrm{H} & 1.60760 & -1.72080 & 0.51520 \\ \mathrm{H} & 1.68500 & -1.77190 & -1.24380 \\ \mathrm{C} & -6.77420 & 1.25460 & -0.43100 \\ \mathrm{H} & -6.61190 & 1.18240 & -1.50990 \\ \mathrm{H} & -7.84160 & 1.22320 & -0.22620 \\ \mathrm{H} & -6.36500 & 2.20060 & -0.06640 \\ \mathrm{C} & 5.60310 & 0.10260 & -0.24130 \\ \mathrm{~S} & 4.32350 & -1.14990 & -0.62060 \\ \mathrm{C} & 2.79140 & -0.15870 & -0.37350 \\ \mathrm{C} & 2.88770 & 0.63690 & 0.91630 \\ \mathrm{~N} & 3.95330 & 1.45950 & 0.97150 \\ \mathrm{C} & 4.98590 & 1.47910 & -0.04410 \\ \mathrm{H} & 6.30950 & 0.12560 & -1.07020 \\ \mathrm{H} & 6.12740 & -0.20920 & 0.66130 \\ \mathrm{H} & 2.69640 & 0.53650 & -1.20850 \\ \mathrm{O} & 2.06540 & 0.53940 & 1.81960 \\ \mathrm{H} & 5.75570 & 2.18150 & 0.27170 \\ \mathrm{H} & 4.58420 & 1.84820 & -0.99100 \\ \mathrm{H} & 4.09110 & 1.97110 & 1.82870\end{array}$


The fit between calculated and experimental infra-red (IR) and VCD data is shown in Figure S4. There is good agreement between calculated and experimental IR spectra, with small differences between calculated and experimental frequencies noted for the carbonyl peaks around $1700 \mathrm{~cm}^{-1}$. There is very good agreement between the experimental VCD spectrum for compound $\mathbf{2 3}$ and the calculated VCD spectrum for the $(S)$ enantiomer.

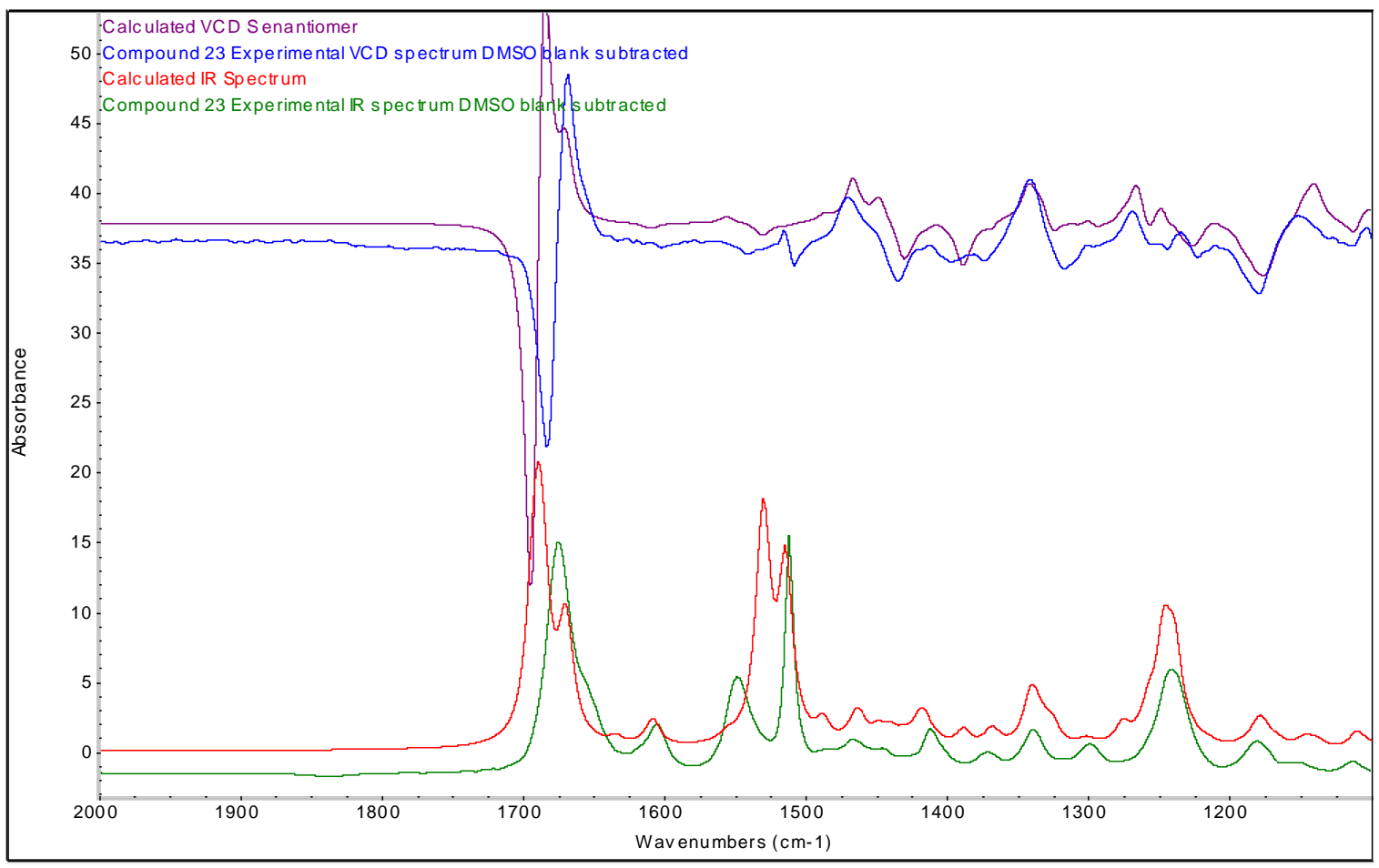

Figure S4. Comparison of calculated infra-red and VCD spectra for the $(S)$ enantiomer (red and purple) with the experimental data for compound $\mathbf{2 3}$ (green and blue). The blank spectra recorded for DMSO-d6 have been subtracted from both experimental spectra. 


\section{References}

1. Vonrhein, C.; Flensburg, C.; Keller, P.; Sharff, A.; Smart, O.; Paciorek, W.; Womack, T.; Bricogne, G. Data processing and analysis with the autoPROC toolbox. Acta Crystallogr. D. Biol. Crystallogr. 2011, 67 (Pt 4), 293-302.

2. Iverson, C.; Larson, G.; Lai, C.; Yeh, L. T.; Dadson, C.; Weingarten, P.; Appleby, T.; Vo, T.; Maderna, A.; Vernier, J. M.; Hamatake, R.; Miner, J. N.; Quart, B. RDEA119/BAY 869766: a potent, selective, allosteric inhibitor of MEK1/2 for the treatment of cancer. Cancer Res. 2009, 69 (17), 6839-6847.

3. Bricogne, G.; Blanc, E.; Brandl, M.; Flensburg, C.; Keller, P.; Paciorek, W.; Roversi, P.; Sharff, A.; Smart, O. S.; Vonrhein, C.; Womack, T. O. 2017. BUSTER version 2.11.7. Cambridge, United Kingdom: Global Phasing Ltd.

4. Emsley, P.; Lohkamp, B.; Scott, W. G.; Cowtan, K., Features and development of Coot. Section Title: Biochemical Methods 2010, 66 (4), 486-501.

5. Wlodek, S.; Skillman A. G.; Nicholls A. Automated ligand placement and refinement with a combined force field and shape potential. Acta Cryst D. Biol. Crystallogr. 2006, 62, 741-749.

6. The PyMOL Molecular Graphics System, Version 1.7: Schrödinger, LLC, New York, NY, 2010.

7. Laskowski, R. A.; MacArthur, M. W.; Moss, D. S.; Thornton, J. M. PROCHECK - a program to check the stereochemical quality of protein structures. J. App. Cryst. 1993, 26, 283-291.

8. Gaussian 16, Revision B.01. M. J. Frisch, G. W. Trucks, H. B. Schlegel, G. E. Scuseria, M. A. Robb, J. R. Cheeseman, G. Scalmani, V. Barone, G. A. Petersson, H. Nakatsuji, X. Li, M. Caricato, A. V. Marenich, J. Bloino, B. G. Janesko, R. Gomperts, B. Mennucci, H. P. Hratchian, J. V. Ortiz, A. F. Izmaylov, J. L. Sonnenberg, D. Williams-Young, F. Ding, F. Lipparini, F. Egidi, J. Goings, B. Peng, A. Petrone, T. Henderson, D. Ranasinghe, V. G. Zakrzewski, J. Gao, N. Rega, G. Zheng, W. Liang, M. Hada, M. Ehara, K. Toyota, R. Fukuda, J. Hasegawa, M. Ishida, T. Nakajima, Y. Honda, O. Kitao, H. Nakai, T. Vreven, K. Throssell, J. A. Montgomery, Jr., J. E. Peralta, F. Ogliaro, M. J. Bearpark, J. J. Heyd, E. N. Brothers, K. N. Kudin, V. N. Staroverov, T. A. Keith, R. Kobayashi, J. Normand, K. Raghavachari, A. P. Rendell, J. C. Burant, S. S. Iyengar, J. Tomasi, M. Cossi, J. M. Millam, M. Klene, C. Adamo, R. Cammi, J. W. Ochterski, R. L. Martin, K. Morokuma, O. Farkas, J. B. Foresman, D. J. Fox, Gaussian, Inc., Wallingford CT, 2016. 